\title{
O ESTUDO DO LOOPING VERTICAL DE UM BALDE COM ÁGUA COM APLICATIVO DE VIDEOANÁLISE PARA DISPOSITIVOS MÓVEIS
}

\author{
FRANCISCO ADEIL GOMES DE ARAÚJO* \\ Secretaria de Educação Básica do Ceará- SEDUC \\ EEMTI Estado do Amazonas, Bela Vista, Fortaleza-CE CEP 60441-750 \\ MEIRIVÂNI MENESES DE OLIVEIRA \\ Secretaria de Educação Básica do Ceará- SEDUC \\ Av. Gen. Afonso Albuquerque Lima, Cambeba, Fortaleza - CE CEP: 60822-325 \\ ELONEID FELIPE NOBRE \\ Universidade Federal do Ceará - UFCE Campus do Pici, BL 902 \\ Av. Mister Hull, s/n - Pici, Fortaleza - CE, 60455-760 \\ ALEXANDRE GONÇALVES PINHEIRO \\ Faculdade de Educação, Universidade Estadual do Ceará- UECE, \\ Planalto Universitário, Quixadá-CE, CEP: 63.900-000 \\ MARCONY SILVA CUNHA \\ Departamento de Física, Universidade Estadual do Ceará - UECE \\ Av. Dr. Silas Munguba,1700, Itaperi, 60714-903, Fortaleza-CE
}

\begin{abstract}
Resumo
O presente trabalho apresenta uma proposta de como explorar o looping vertical de um balde com água utilizando o aplicativo de videoanálise NewtonDV. Com a análise do vídeo do movimento foi possivel verificar a força exercida pelo balde sobre a água no topo da trajetória circular, o menor valor da velocidade tangencial que mantém a água no balde e a força exercida pelo balde sobre a água no ponto mais baixo de sua trajetória. A utilização deste aplicativo de videoanálise permite estabelecer uma interação entre a teoria e a aplicação do conhecimento físico a situações concretas, possibilitando o aprendizado com diferentes representações como gráficos, diagramas, tabelas, imagens e o próprio vídeo. Desta maneira, espera-se que este trabalho norteie a ação pedagógica do professor, fazendo com que os smartphones/tablets sejam vistos como recursos didáticos para as aulas de Física.
\end{abstract}

Palavras-chave: Movimento Circular; ensino de Física; videoanálise; dispositivos móveis.

\footnotetext{
*E-mail: chicoadeil@gmail.com
} 


\begin{abstract}
The present work presents a proposal of how to explore the vertical looping of a bucket with water using NewtonDV videoanalysis application for mobile devices. With the videoanalysis of the movement, it was possible to verify the force exerted by the bucket on the water at the top of the circular trajectory, also the smaller value of the tangential velocity that keeps the water in the bucket and the force exerted by the bucket on the water at the lowest point of their trajectory. The use of this videoanalysis application allowed to establish an interaction between theory and the application of physical knowledge to concrete situations, enabling learning with different representations such as graphs, diagrams, tables, images and the video itself. In this way, this work is expected to guide the pedagogical action of the teacher, making the smartphones/tablets be seen as a didactic resource for the physics classes.
\end{abstract}

Keywords: Circular Motion; Physics teaching; Videoanalysis; mobile devices.

\title{
1 INTRODUÇÃO
}

A videoanálise de objetos em movimento é explorada nas aulas de Física (LAWS \& PFISTER, 1988) e Matemática (OLDKNOW, 2003) há vários anos. Sua vantagem reside no fato de que medidas de posições feitas por aplicativos apropriados tendem a ter menor incerteza relativa do que outros tipos de medição feitas em laboratórios. As distâncias são medidas em pixels e em seguida, dimensionadas em metros usando como comparação algum objeto de comprimento conhecido encontrado na imagem (LAWS \& PFISTER, 1988). Desta forma pode-se obter gráficos, diagramas, tabelas e imagens estroboscópicas do movimento.

Hoje a maioria dos jovens possui um dispositivo móvel (smartphone/tablet) e, devido aos inúmeros sensores disponíveis nesses dispositivos, pode-se dizer que eles carregam um laboratório móvel em seus bolsos. Aproveitando-se disso, recentemente muitos exemplos do uso de dispositivos móveis como ferramentas experimentais para realizar um estudo quantitativo de movimentos bidimensionais foram publicados (HIRTH et al, 2016, KLEIN et al 2014, 2015a, 2015b, SADEK, 2015, OLDKNOW et al, 2010, ARAÚJO et al, 2017).

Neste trabalho descreve-se uma atividade experimental na qual um balde contendo água foi girado em um plano vertical. O movimento foi filmado e o vídeo foi analisado com o aplicativo NewtonDV ${ }^{1,2}$, disponível para o sistema operacional iOS. Outros aplicativos podem ser utilizados, como o Video Physics ${ }^{3}$ e o Graphical Analysis ${ }^{4}$ também disponíveis para iOs, os aplicativos VidAnalysis ${ }^{5}$ e Lablet $^{6}$, para o sistema

\footnotetext{
${ }^{1}$ https://itunes.apple.com/br/app/newtondv/id717653395?mt=8

${ }^{2}$ Tutorial do aplicativo NewtonDV: https://www.youtube.com/watch?v=Gv7jZUByTXc

${ }^{3} \mathrm{https}$ ://itunes.apple.com/br/app/vernier-video-physics/id389784247?mt=8

${ }^{4} \mathrm{https} / / /$ itunes.apple.com/br/app/vernier-graphical-analysis/id522996341?mt=8

${ }^{5}$ https://play.google.com/store/apps/details?id=com.vidanalysis.free

${ }^{6} \mathrm{https} / / /$ play.google.com/store/apps/details?id=nz.ac.auckland.lablet\&hl=en
} 


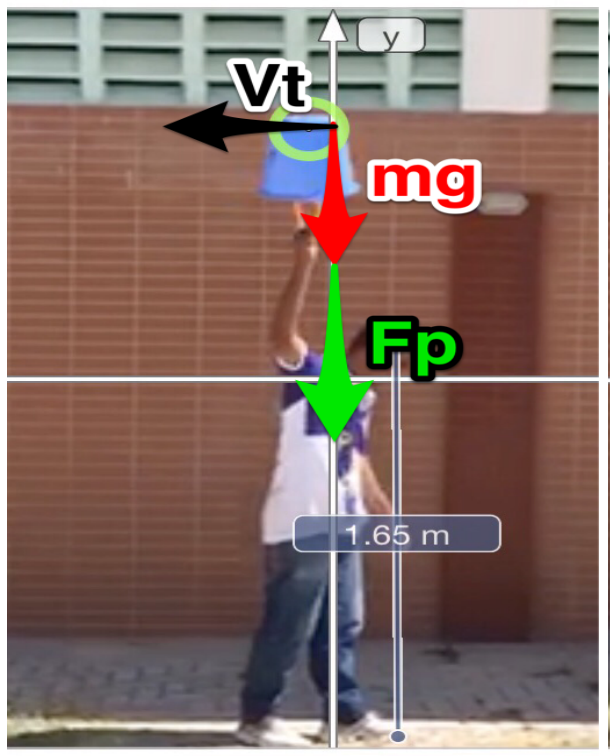

$00: 01.45$

(a) Forças sobre o sistema e a cidade tangencial com o balde no topo da trajetória circular, instante $t=1: 45 \mathrm{~s}$. Imagem gerada pelo aplicativo NewtonDV, 2017.

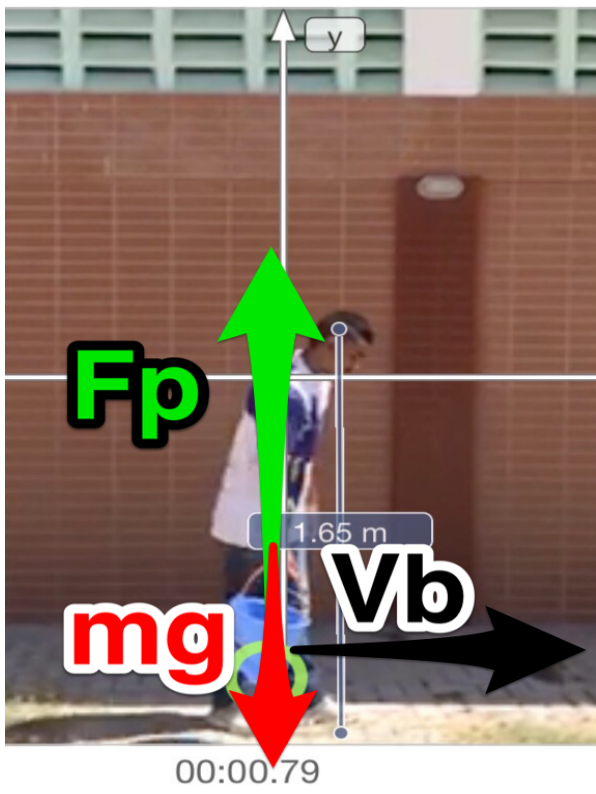

(b) Forças sobre o sistema no ponto mais baixo de sua trajetória, instante $t=0,79$ s. Imagem gerada pelo aplicativo NewtonDV, 2017.

operacional Android e o software de utilização online Web Tracker ${ }^{7}$.A partir dos dados coletados podese investigar a força exercida pelo balde sobre a água no topo da trajetória circular, o menor valor da velocidade tangencial que mantém a água no balde e a força exercida pelo balde sobre a água no ponto mais baixo de sua trajetória.

O presente trabalho está dividido em cinco seções, sendo esta a primeira. Na segunda seção será apresentada uma revisão teórica do looping do balde com água. Na terceira, apresenta-se os procedimentos necessários para a captura de vídeo. Na quarta, são apresentados os resultados do experimento e as repostas para as questões formuladas na segunda seção. Na quinta e última seção, são discutidos alguns pontos importantes apresentados neste trabalho.

\section{REVISÃO TEÓRICA - O LOOPING DO BALDE COM ÁGUA}

Nesta seção é apresentada uma breve revisão teórica, buscando responder às seguintes perguntas: Qual a força exercida pelo balde sobre a água no topo da trajetória circular? Qual o menor valor de velocidade tangencial, $v_{t}$, que mantém a água no balde? Como mostra a Figura 1a. Qual a força exercida pelo balde sobre a água no ponto mais baixo de sua trajetória, onde a velocidade do balde é $v_{b}$ ? Como mostra a Figura 1b.

\footnotetext{
${ }^{7}$ https://www.splab.io/wt/
} 
Durante o movimento do balde as únicas forças que atuam no topo da trajetória são a força que o balde faz sobre a água, $F_{P}$, e o peso, m.g, como pode-se observar na Figura 1a. Aplicando-se a segunda lei de Newton à água quando ela passa pelo topo da trajetória circular, tem-se que

$$
\sum F_{y}=m a_{y}
$$

Sendo $P=m g, a_{y}=v_{t}^{2} / r$, tem-se que, no ponto mais alto da trajetória,

$$
F_{P}+m g=m \frac{v_{t}^{2}}{r}
$$

Assim a força que o balde faz na água depende da sua massa, da sua velocidade no topo e do raio da trajetória, como mostra a equação (3).

$$
F_{P}=m\left(\frac{v_{t}^{2}}{r}-g\right)
$$

$\mathrm{Na}$ iminência para a água cair do balde ocorre que a força que o balde exerce sobre a água, $F_{P}$, é igual à zero.

$$
\frac{v_{t, \min }^{2}}{r}-g=0
$$

Dessa forma, isolando o $v_{t, \min }$ na equação (4), tem-se

$$
v_{t, \min }=\sqrt{g r}
$$

Este, portanto, é o valor crítico de velocidade, abaixo do qual a água deve cair.

Por último, aplicando-se a segunda lei de Newton à água quando ela passa pelo ponto mais baixo de sua trajetória circular com velocidade $v_{b}$, tem-se que

$$
F_{P}-m g=m \frac{v_{b}^{2}}{r}
$$

Assim, obtém-se

$$
F_{P}=m\left(\frac{v_{b}^{2}}{r}+g\right) .
$$

Nas próximas seções será descrita a videoanálise do experimento que permitiu encontrar as respostas para as questões formuladas.

\section{EXECUÇÃO DO EXPERIMENTO}

$\mathrm{Na}$ execução do experimento é preciso alguns cuidados para que os resultados sejam consistentes. Primeiramente, ao gravar o movimento, é importante manter o dispositivo imóvel, perpendicular ao plano do movimento e não seguir o objeto. Outro detalhe importante é que deve existir algum objeto de dimensões conhecidas no vídeo e este deve estar no mesmo plano do movimento (LAWS \& PFISTER, 1988, OLDKNOW et al, 2010), como por exemplo a altura do estudante na Figura 2. 


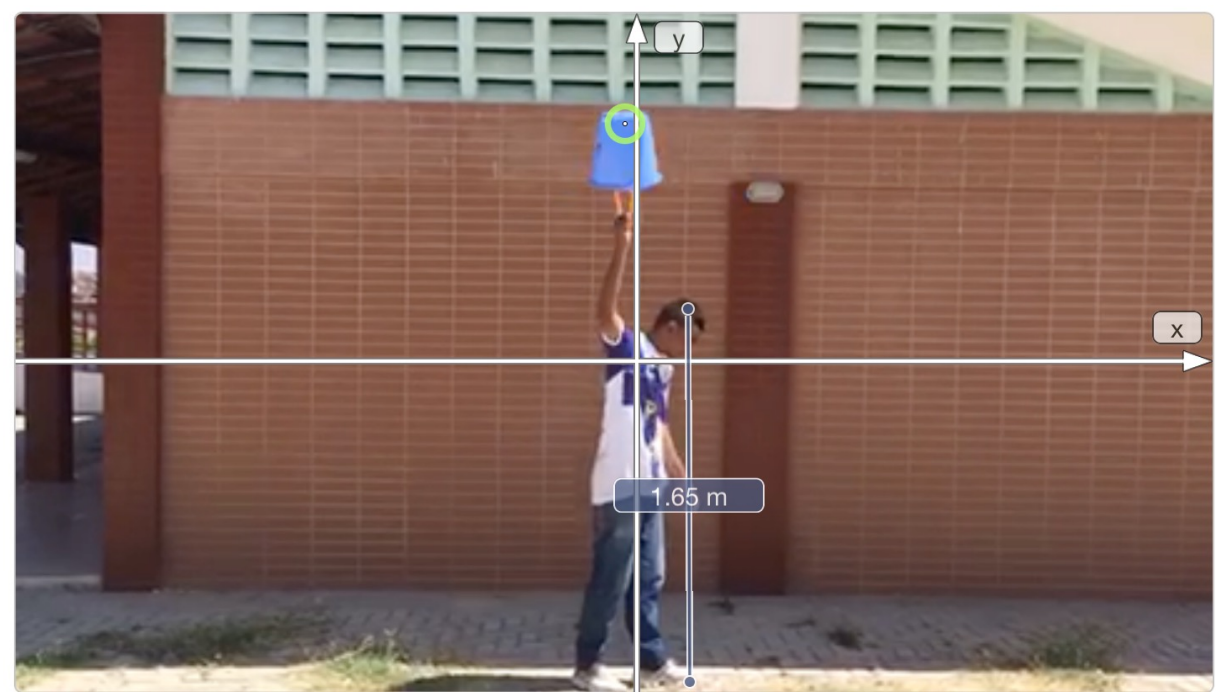

Figura 2: Câmera perpendicular ao plano do movimento.

\section{RESULTADOS}

Após marcar manualmente os pontos por onde o balde contendo água passou, o aplicativo gera alguns gráficos, como o da posição pelo tempo. Na Figura 3, percebe-se que este movimento se trata de um Movimento Harmônico Simples (MHS).

A partir da função de regressão sinusoidal $y=a \sin (b t+c)+d$ gerada pelo aplicativo tem-se o valor de cada coeficiente da função que melhor representa o movimento do balde em trajetória circular. Como se pode observar na equação

$$
y=1,13(\mathrm{~m}) \cdot \sin (4,69(\mathrm{rad} / \mathrm{s}) t+1,26(\mathrm{rad}))+(-0,06)(\mathrm{m})
$$

O coeficiente a, dado em metros, representa a"amplitude" da função, ou seja, o raio da trajetória. O coeficiente $b$, dado em radianos/s, representa a frequência angular da função que pode ser calculado pela expressão $2 \pi / T$. O coeficiente $c$, dado em radianos, representa a constante de fase, ele desloca a curva para a esquerda ou para direita. Já o coeficiente $d$, dado em metros, representa o "deslocamento" da curva para cima ou para baixo. O coeficiente $d$ indica o quanto a origem das coordenadas se distancia da origem da oscilação. Se a origem estiver no centro da oscilação, o coeficiente $d$ deve ser zero. A Figura 4 reforça a relação existente entre o movimento circular e o movimento harmônico simples (MHS) fato que pode ser explorado pelo professor durante a atividade.

O aplicativo também gera uma tabela com dados sobre a posição, a velocidade e a aceleração do balde durante sua trajetória circular, como pode-se observar na Figura 5. O valor da velocidade do balde no instante $0,79 \mathrm{~s}$ em que ele se encontra no ponto mais baixo da trajetória é $v_{b}$, cujo o valor é de aproximadamente $6 \mathrm{~m} / \mathrm{s}$ enquanto que a velocidade do balde no ponto mais alto da trajetória, no instante $1,46 \mathrm{~s}$, é $v_{t}$, de valor é aproximadamenteigual a $4 \mathrm{~m} / \mathrm{s}$. Com essas informações e considerando que a água contida no balde durante o looping tinha aproximadamente a massa de $1 \mathrm{~kg}$, pode-se responder às perguntas propostas na seção 2 . 


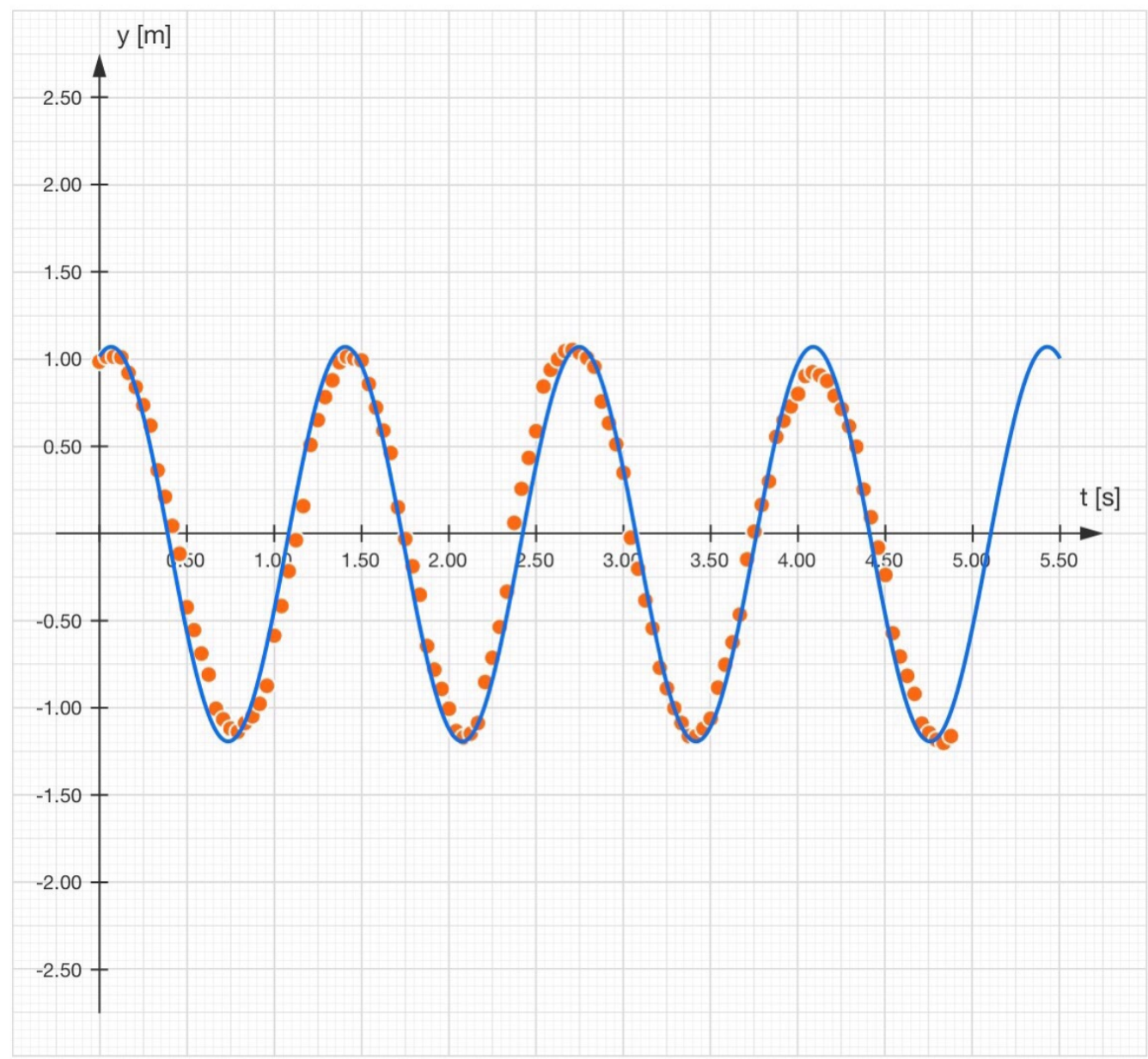

Trend curve

\begin{tabular}{|c|c|c|c|c|}
\hline None & Linear & Quadratic & Sine & Sine (damped) \\
\hline
\end{tabular}

$y=\begin{array}{ccccc}1.13 & \cdot \sin ( & 4.69 & \cdot t & +1.26 \\ a & b & c & -0.06\end{array}$

Figura 3: Diagrama da posição na direção y pelo tempo com uma curva de tendência trigonométrica. Fonte:Imagem gerada pelo aplicativo NewtonDv durante a realização do experimento, 2017. 


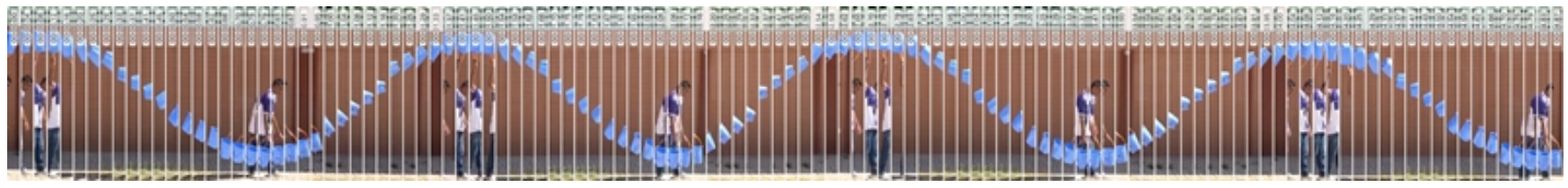

Figura 4: Série de imagens horizontais que mostram a relação entre o movimento circular e o Movimento Harmônico Simples (MHS) Fonte:Imagem gerada pelo aplicativo NewtonDV durante a realização do experimento, 2017.

\begin{tabular}{|c|c|c|c|c|c|c|c|c|}
\hline \multirow{3}{*}{$\begin{array}{l}\text { iPad } ~ \\
\frac{\text { Time }}{2} \\
{[s]}\end{array}$} & \multicolumn{5}{|c|}{$21: 49$} & \multicolumn{3}{|r|}{$42 \%$} \\
\hline & \multicolumn{2}{|c|}{ Position } & \multicolumn{3}{|c|}{ Velocity } & \multicolumn{3}{|c|}{ Acceleration } \\
\hline & $\begin{array}{c}X \\
{[\mathrm{~m}]}\end{array}$ & $\begin{array}{c}y \\
{[\mathrm{~m}]}\end{array}$ & $\begin{array}{c}\mathbf{V} \\
{[\mathrm{m} / \mathrm{s}]}\end{array}$ & & & $\underset{\left[\mathrm{m} / \mathrm{s}^{2}\right]}{\mathrm{a}}$ & $\underset{\left[\mathrm{m} / \mathrm{s}^{2}\right]}{a x}$ & $\underset{\left[\mathrm{m} / \mathrm{s}^{2}\right]}{\mathrm{ay}}$ \\
\hline 0.79 & -0.063 & -1.226 & 6.033 & 2 & 1.252 & 37.043 & -36.480 & -6.438 \\
\hline 0.83 & 0.183 & -1.174 & 4.490 & 4.381 & 0.984 & 21.134 & -8.583 & 19.313 \\
\hline 0.88 & 0.365 & -1.133 & 4.403 & 4.024 & 1.788 & 20.357 & -6.438 & 19.313 \\
\hline 0.92 & 0.533 & -1.058 & 4.564 & 3.756 & 2.593 & 126.732 & 60.084 & 111.584 \\
\hline 0.96 & 0.689 & -0.950 & 9.573 & 6.259 & 7.243 & 128.554 & -107.292 & -70.813 \\
\hline 1.00 & 0.950 & -0.648 & 4.650 & 1.788 & 4.292 & 17.300 & -2.146 & 17.167 \\
\hline 1.04 & 1.025 & -0.469 & 5.288 & 1.699 & 5.007 & 42.756 & -40.771 & -12.875 \\
\hline 1.08 & 1.095 & -0.261 & 4.471 & 0.000 & 4.471 & 15.174 & -10.729 & 10.730 \\
\hline 1.13 & 1.095 & -0.075 & 4.938 & -0.447 & 4.918 & 113.080 & -62.230 & 94.417 \\
\hline 1.17 & 1.077 & 0.130 & 9.360 & -3.040 & 8.852 & 126.678 & 4.292 & -126.605 \\
\hline 1.21 & 0.950 & 0.499 & 4.580 & -2.861 & 3.577 & 7.737 & -4.291 & -6.438 \\
\hline 1.25 & 0.831 & 0.648 & 4.493 & -3.040 & 3.308 & 25.025 & -12.875 & -21.458 \\
\hline 1.29 & 0.704 & 0.786 & 4.315 & -3.577 & 2.414 & 81.655 & -81.542 & 4.292 \\
\hline 1.33 & 0.555 & 0.887 & 7.441 & -6.975 & & 72.102 & 57.938 & -42.917 \\
\hline 1.38 & 0.265 & 0.995 & 4.631 & & 0.805 & 33.519 & 21.458 & -25.750 \\
\hline 1.42 & 0.075 & 1.028 & 3.676 & & -0.268 & 8.583 & -8.583 & 0.000 \\
\hline 1.46 & -0.078 & 1.017 & 4.033 & -4.024 & -0.268 & 138.087 & -115.876 & -75.105 \\
\hline & & & & & & & & $\complement^{\Uparrow}$ \\
\hline & & & able & $\varliminf_{\text {Diagram }}$ & Series & & ? & \\
\hline
\end{tabular}

Figura 5: Tabela com dados sobre posição velocidade e aceleração. Fonte: Imagem gerada pelo aplicativo NewtonDV, 2017 
Para isso, deve-se substituir os valores apresentados na Figura 5 nas equações (3) e (7). Assim, obtém-se:

$$
F_{p}=m\left(\left(v_{t}^{2}\right) / r-g\right) \rightarrow F_{p}=1 .\left(4^{2} / 1,13-9,8\right)=4,36 N,
$$

e

$$
F_{p}=m\left(\left(v_{b}^{2}\right) / r+g\right) \rightarrow F_{p}=1 .\left(6^{2} / 1,13+9,8\right)=41,65 \mathrm{~N} .
$$

$\mathrm{O}$ valor da força que o balde faz sobre a água no topo da trajetória é de $4,36 \mathrm{~N}$, enquanto que no ponto inferior é de 41,65 $\mathrm{N}$. No ponto mais baixo da trajetória o valor da força do balde sobre a água deve ser maior do que o peso para que seja gerada uma resultante na direção radial para cima.

$\mathrm{O}$ menor valor da velocidade tangencial que mantém a água no balde pode ser encontrado substituindo os valores do raio da trajetória e da aceleração da gravidade na equação (5).

$$
v_{t, \min }=\sqrt{g r}=\sqrt{9,8 \times 1,13}=3,32 \mathrm{~m} / \mathrm{s} .
$$

A tabela mostra que no ponto mais alto da trajetória a velocidade $v_{t}$ é de aproximadamente $4 \mathrm{~m} / \mathrm{s}$ superior a de $3,32 \mathrm{~m} / \mathrm{s}$ previstos na equação (11).

\section{CONSIDERAÇÕES FINAIS}

O presente trabalho propõe a realização de experimentos de baixo custo para explorar o estudo do movimento circular de maneira que os alunos possam fazer uso de seus próprios dispositivos móveis, para gravar um vídeo e analisá-lo.

O uso de materiais de baixo custo para a realização de experimentos com o auxílio de aplicativos para smartphones/tablets pode contribuir para explorar conteúdos da Física e da Matemática, mesmo que a escola não possua um laboratório adequado, além de aumentar a autonomia e promover a motivação entre os estudantes.

Outro ponto é que o aplicativo utilizado neste trabalho não requer o uso contínuo da internet, já que, após a sua instalação, seu uso pode ser totalmente off-line, o que contribui para que todos os alunos possam explorá-lo independente da velocidade da internet na escola.

Espera-se com este artigo que o leitor venha a se sentir motivado a realizar investigações de acontecimentos do mundo real com a ajuda de aplicativos de videoanálise para dispositivos móveis tão presentes em nosso dia-a-dia.

\section{Referências}

[1] LAWS,P.; PFISTER,H. Using Digital Video Analysis in Introductory Mechanics Projects. The Physics Teacher. 1998. Disponível em: <https://cienciaescolar.files.wordpress.com/2009/12/usandoanalisis-video-digital-en-proyectos-de-fisica.pdf $>$,1998. 36. 282-287. Último acesso em: 17 de fevereiro de 2018.

[2] OLDKNOW, A. Mathematics from still and video images. Micromath Summer.2003. Disponível em: <https://www.mah.se/pages/74006/video1.pdf>. 30-34. Último acesso em: 17 de fevereiro de 2017. 
[3] HIRTH, M; KUHN, J; MÜLLER, A; ROHS, M; KLEIN, P. iMobilePhysics: Seamless Learning durch Experimente mit Smartphones \& Tablets in Physik. Disponível em:<https://www.researchgate.net/publication/308723033_iMobilePhysics_Seamless_Learning _durch_Experimente_mit_Smartphones_Tablets_in_Physik>, 2016. Último acesso em: 17 de fevereiro de 2018.

[4] KLEIN,P.;GRÖBER,S.; KUHN,J.;WILHELM,T. Bewegungen mit der App Video Analysis analysieren. Smart Physik, Disponível em: $<$ http://onlinelibrary.wiley.com/doi/10.1002/piuz.201590033/abstract>,2015. Último acesso em: 17 de fevereiro de 2018.

[5] KLEIN,P.;GRÖBER,S.; KUHN,J ; MÜLLER, A. Video analysis of projectile motion using tablet computers as experimental tools. Disponível em: <http://iopscience.iop.org/article/10.1088/00319120/49/1/37/meta>, 2014. Último acesso em: 17 de fevereiro de 2018.

[6] SADEK, R. VidAnalysis - Videoanalyse für Android. Disponível em: $<$ http://pluslucis.univie.ac.at/PlusLucis/151/S45.pdf>,2015. Último acesso em: 17 de fevereiro de 2018.

[7] KLEIN, P; KUHN, J.; MÜLLER, A. Mobile Videoanalyse - Wurf vom fahrenden Skateboard. Naturwissenschaften im Unterricht Physik: Materialien \& Methoden. Experimentieren mit Smartphone und Tablet. 145, 24-26 (2015a).

[8] KLEIN, P; KUNH, J; MÜLlER, A.; GRÖBER, S., Video Analysis Exercises in regular Introductory Physics Courses: Effects of Conventional Methods and Possibilities of Mobile Devices, p. 270-288, Multidisciplinary Research on Teaching and Learning, 2015b. Disponível em:<https://link.springer.com/chapter/10.1057/9781137467744_15>. Acesso: 17 de fevereiro de 2018.

[9] OLDKNOW, A; TAYLOR, R; TETLOW, L. Teaching Mathematics Using ICT. Continuum International Publishing Group. 3rd Edition (2010).

[10] ARAÚJO, F.A.G; OLIVEIRA, M.M.; NOBRE, E.F.; PINHEIRO, A.G.; CUNHA, M.S. Estudo do Movimento com o Aplicativo VidAnalysis: Possibilidades no Estudo de Lançamento de projéteis. Rev Prof de Física. 1, n.2 (2017). Disponível em: $<$ http://periodicos.unb.br/index.php/rpf/article/view/25088/19826>. Acesso em: 17 de fevereiro de 2018. 